\title{
HETEROCYCLES 46. SYNTHESIS, CHARACTERIZATION AND BIOLOGICAL EVALUATION OF THIAZOLO[3,2-b][1,2,4]TRIAZOLES BEARING BENZENESULFONAMIDE MOIETY
}

\author{
ANAMARIA CRISTINA ${ }^{1,2}$, DENISA LEONTE ${ }^{1}$, LAURIAN VLASE ${ }^{3}$, LÁSZLÓ CSABA BENCZE ${ }^{4}$, \\ SILVIA IMRE $^{5}$, BOGDAN APAN ${ }^{6}$, CRISTINA MOGOȘAN ${ }^{2}$, VALENTIN ZAHARIA ${ }^{1 *}$ \\ 1 “Iuliu Haţieganu” University of Medicine and Pharmacy, Department of Organic Chemistry, 41 Victor Babeș Street, 400012, \\ Cluj-Napoca, Romania \\ 2 "Iuliu Hatieganu” University of Medicine and Pharmacy, Department of Pharmacology, Physiology and Pathophysiology, 6 \\ Louis Pasteur Street, 400349, Cluj-Napoca, Romania \\ 3 "Iuliu Hatieganu" University of Medicine and Pharmacy, Department of Pharmaceutical Technology and Biopharmaceutics, 41 \\ Victor Babeș Street, 400012, Cluj-Napoca, Romania \\ 4 "Babeş-Bolyai" University, Faculty of Chemistry and Chemical Engineering, Biocatalysis and Biotransformation Research \\ Group, 11 Arany János Street, 400028, Cluj-Napoca, Romania \\ 5University of Medicine and Pharmacy Tîrgu Mureș, Department of Analytical Chemistry and Drug Analysis, 38 Gheorghe \\ Marinescu Street, 540139, Târgu Mureș, Romania \\ 6"Iuliu Haţieganu" University of Medicine and Pharmacy, Department of Pharmacology, Toxicology and Clinical \\ Pharmacology, 23 Gheorghe Marinescu Street, 400337, Cluj-Napoca, Romania
}

*corresponding author: vzaharia@umfcluj.ro

Manuscript received: May 2018

\begin{abstract}
In order to synthesize novel anti-inflammatory and analgesic compounds with reduced ulcerogenic risk, a series of thiazolo[3,2-b][1,2,4]triazoles (6a-6d) and their corresponding acyclic intermediates (5a-5d) bearing benzenesulfonamide moiety were obtained and characterized by spectral analysis $\left({ }^{1} \mathrm{H} N M R,{ }^{13} \mathrm{C}\right.$ NMR, IR and MS). All synthesized compounds were evaluated in vivo for their anti-inflammatory and antinociceptive activities in a rat model of acute inflammation induced by $\lambda$-carrageenan. The compounds $\mathbf{5 b}, \mathbf{5 c}$ and $\mathbf{6 d}$ showed significant anti-inflammatory activity when compared to negative control group, but they did not show superior anti-inflammatory activity when compared to diclofenac, as reference drug. The compounds were also screened for antinociceptive activity in a model of inflammatory hyperalgesia and compounds $\mathbf{5 a}, \mathbf{5 b}$, $\mathbf{5 c}, \mathbf{6 a}, \mathbf{6 d}$ presented a significant increase of nociceptive threshold in the inflamed paw. Moreover, compounds $5 \mathbf{c}, 6 \mathbf{6 a}, \mathbf{6 b}, \mathbf{6 c}$ and $\mathbf{6 d}$ did not show any significant ulcerogenic activity.
\end{abstract}

\section{Rezumat}

$\mathrm{Cu}$ scopul de a obține noi molecule cu potențial antiinflamator și analgezic, dar cu risc ulcerogen redus, au fost sintetizați și caracterizați spectral $\left({ }^{1} \mathrm{H}\right.$ RMN,${ }^{13} \mathrm{C}$ RMN, IR și MS) o serie de derivați tiazolo[3,2-b][1,2,4]triazolici (6a-6d) și intermediarii lor aciclici (5a-5d). Compușii sintetizați au fost evaluați in vivo pentru activităţile lor antiinflamatoare și antinociceptive utilizând un model experimental de inflamație acută indusă de $\lambda$-caragenan la şobolan. Compuşii $\mathbf{5 b}, \mathbf{5 c}$ şi $\mathbf{6 d}$ au prezentat o activitate antiinflamatoare semnificativă comparativ cu grupul control negativ, dar nu superioară diclofenacului, medicamentul de referință. Compușii obținuți au fost de asemenea supuşi unui screening pentru activitatea antinociceptivă utilizând un model de hiperalgezie inflamatorie, în care derivații $5 \mathbf{a}, \mathbf{5 b}, \mathbf{5 c}, \mathbf{6 a}, \mathbf{6 d}$ au prezentat o creștere semnificativă a pragului nociceptiv. În plus, compuşii $5 \mathbf{c}, \mathbf{6 a}, \mathbf{6 b}, \mathbf{6 c}$ şi $\mathbf{6 d}$ nu au prezentat activitate ulcerogenă semnificativă.

Keywords: thiazolo[3,2-b][1,2,4]triazole; anti-inflammatory activity; antinociceptive activity; ulcerogenic activity

\section{Introduction}

Non-steroidal anti-inflammatory drugs (NSAIDs) constitute an important pharmacological class of drugs used for the treatment of pain and inflammation [31]. These drugs inhibit prostaglandin-endoperoxide $\mathrm{H}$ synthase (PGHS) or cyclooxygenase (COX), in a nonselective or selective manner $[3,19,36]$. Despite the excellent anti-inflammatory and analgesic effects of NSAIDs, gastrointestinal toxicity associated with the long-term use of these drugs proved to be a major drawback [21]. To limit the risk of gastrointestinal side effects induced by NSAIDs, a great progress has been made toward developing selective COX-2 inhibitors where they exhibited anti-inflammatory and analgesic activities comparable to those of nonselective COX inhibitors but with least gastrointestinal damage [22]. It has also been shown the importance of sulfonamide group in the anti-inflammatory/analgesic activities [15]. Compounds with sulfonamide group in their structure presented increased COX-2 selectivity, 
FARMACIA, 2018, Vol. 66, 5

because of the presence of "side pocket" in the structure of COX-2 enzymes where sulfonamide group can easily fit. The structure of COX-2 is different than the structure of COX-1 with a single amino-acid change from the comparatively bulky isoleucine (Ile) in COX-1 to valine at position 523 in COX-2 (equivalent to position 509 in COX-1). The conformational changes produced by this change of amino-acids, resulted in enhanced access to a "side pocket" that allowed the binding of COX-2-specific inhibitors by providing a docking site for the bulky phenylsulfonamide [13]. Besides, the compounds with sulfonamide group in their structures have antiulcer potential [7], which represents an advantage comparing to classical NSAIDs. Recent studies revealed that the compounds containing various heterocycles like 1,2,4-triazole and condensed heterocycles are good pharmacophores for anti-inflammatory and analgesic activities [33, 39]. $1,2,4$-Triazole ring is found in the structure of many drugs, as well as other several drug candidates presenting anti-inflammatory [26], analgesic [1], antioxidant [34], antimicrobial [32] and anticonvulsant [29] properties. The 1,3-thiazole ring is an important pharmacophore with anti-inflammatory and analgesic properties [24, 27]. The condensation of 1,2,4-triazole and 1,3-thiazole in the same molecule, could lead to novel compounds with superior pharmacological activity due to the biological potential of each system and to the reciprocal influence between the two heterocyclic rings. Thiazolo[3,2-b][1,2,4]triazole fused ring system having various substituents is found in diverse compounds with biological activities like antimicrobial [5], analgesic, anti-inflammatory [38, 39, 43], anticancer [20] and antioxidant [2]. Based on these findings and in continuation of our interest towards pharmacological properties of thiazolo[3,2-b][1,2,4]triazole derivatives, our aim in the present work is the synthesis, characterization and pharmacological evaluation of some thiazolo[3,2-b][1,2,4]triazole derivatives and their corresponding acyclic intermediates bearing benzenesulfonamide moiety.

\section{Materials and Methods}

\section{Chemistry}

All chemical reagents and solvents necessary for the synthesis were obtained from Sigma-Aldrich Chemie GmbH (Steinheim, Germany), TCI Europe N.V. (Zwijndrecht, Belgium) or Merck KgaA (Darmstadt, Germany) and were used as supplied, without any purification.

Thin layer chromatography (TLC) was performed using Merck Kieselgel 60F254 sheets (Darmstadt, Germany), and a mixture of dichloromethane:acetone $2: 1 \mathrm{v} / \mathrm{v}$ as elution system. The spots were viewed in UV light at $254 \mathrm{~nm}$. The compounds were recrystallized from ethanol or a mixture of ethanol:water. Preparative chromatographic purifications were performed using
Merck Kieselgel $60 \AA$ column chromatography and a mixture of dichloromethane:acetone $2: 1 \mathrm{v} / \mathrm{v}$ as eluent. Melting points were determined with an Electrothermal IA 9000 digital apparatus (Bibby Scientific Limited, Staffordshire, UK) using open glass capillaries and were uncorrected.

The MS analyses were recorded using an Agilent 1100 Ion Trap mass spectrometer (Agilent Technologies, USA) operating at $70 \mathrm{eV} .{ }^{1} \mathrm{H}-\mathrm{NMR}$ and ${ }^{13} \mathrm{C}-\mathrm{NMR}$ spectra were recorded on a Bruker Avance DPX-300 spectrometer (Billerica, Massachusetts, USA) operating at $600 \mathrm{MHz}$ and $151 \mathrm{MHz}$, in DMSO and $\mathrm{CDCl}_{3}$ as solvents and tetramethylsilane (TMS) as internal standard. The spectral data are mentioned in Results and Discussion section, using the following abbreviations for peak patterns: s-singlet, d-doublet, dd-double doublet, t-triplet, q-quartet, br s-broad signal.

The FT-IR analysis was performed on a Jasco FT/IR 470 Plus spectrometer (Easton, Maryland, USA) using the ATR (Attenuated Total Reflection) technique. The IR spectra were recorded between 4000 and $400 \mathrm{~cm}^{-1}$ wavelengths at $4 \mathrm{~cm}^{-1}$ resolution.

Synthesis of thiazolo[3,2-b][1,2,4]triazoles and their corresponding thioethers

Synthesis of 4-(hydrazinecarbonyl)benzenesulfonamide 2. To a solution of 4-sulfamoylbenzoic acid ( $1 \mathrm{mmol}, 0.201 \mathrm{~g})$ in $10 \mathrm{~mL}$ absolute methanol, $0.50 \mathrm{~mL}$ of concentrated sulfuric acid was added and the reaction mixture was refluxed for 6 hours. The formed precipitate was filtered and recrystallized by absolute ethanol. The methyl 4-sulfamoylbenzoate 1 previously obtained was dissolved in absolute methanol $(5 \mathrm{~mL})$ and treated with hydrazine hydrate ( $2 \mathrm{mmol}$ ). The mixture was refluxed using a water bath for another 6 hours and the formed precipitate was filtered and recrystallized by absolute ethanol.

Synthesis of 4-(5-thioxo-4,5-dihydro-1H-1,2,4-triazol-3-yl)benzenesulfonamide 4. A suspension of 4(hydrazinecarbonyl)benzenesulfonamide $2(10 \mathrm{mmol}$, $2.150 \mathrm{~g})$, potassium thiocyanate $(30 \mathrm{mmol}, 3 \mathrm{~g})$ and concentrated hydrochloric acid $(10 \mathrm{~mL})$ was refluxed for 3 hours. After cooling, the resulting thiosemicarbazide 3 was removed by filtration and dissolved in $10 \%$ sodium hydroxide solution $(5 \mathrm{~mL})$ and refluxed for 3 hours. The mixture was filtered hot and poured onto ice followed by acidification with concentrated acetic acid to $\mathrm{pH} 5-6$. The resulting precipitate was filtered and recrystallized by absolute ethanol.

General procedure for the synthesis of 4-(5-((2-aryl2-oxoethyl)thio)-1H-1,2,4-triazol-3-yl)benzene-

sulfonamide (5a-5d). A mixture of 4-(5-thioxo-4,5dihydro-1 $H$-1,2,4-triazol-3-yl)benzenesulfonamide 4 (1 mmol, $0.256 \mathrm{~g})$, sodium hydrogencarbonate (1 mmol, $0.084 \mathrm{~g})$ and the corresponding phenacyl bromide $(1.2 \mathrm{mmol})$ was stirred in $10 \mathrm{~mL}$ of absolute ethanol at room temperature for 24 to 48 hours, depending of the reaction progress, monitored by TLC. The formed precipitate was poured onto ice, 
filtered and purified by recrystallization or by column chromatography to remove unwanted products.

General procedure for the synthesis of thiazolo[3,2-b] $[1,2,4]$ triazole derivatives (6a-6d). Route A: $0.100 \mathrm{~g}$ of 4-(5-((2-aryl-2-oxoethyl)thio)-1H-1,2,4-triazol3-yl)benzenesulfonamide 5a-5d previously obtained was poured onto $0.5 \mathrm{~mL}$ of concentrated sulfuric acid for 1 to 12 hours, depending of the cyclization progress which was monitored by TLC. When the reaction was complete, the mixture was poured onto ice and the formed precipitate was filtered and washed with a solution of sodium hydrogen carbonate. The obtained product was purified by recrystallization or by column chromatography to remove unwanted products. Route $B$ : to a solution of 4-(5-thioxo-4,5-dihydro- $1 H$-1,2,4triazol-3-yl)benzenesulfonamide 4 ( $1 \mathrm{mmol}, 0.256 \mathrm{~g}$ ) in $10 \mathrm{~mL}$ absolute ethanol, the corresponding phenacyl bromide was added $(1.2 \mathrm{mmol})$. The resulting reaction mixture was refluxed for 2 hours and $0.5 \mathrm{~mL}$ of concentrated sulfuric acid was added to the mixture, after cooling. The mixture was refluxed for another 2 to 30 hours, depending of the reaction progress, monitored by TLC. When the reaction was complete, the mixture was poured on ice and neutralized with a solution of sodium hydroxide. The precipitate was purified by recrystallization or by column chromatography to remove unwanted products.

\section{Biological evaluation}

Animals. For anti-inflammatory and analgesic activity tests, 10 groups of male Charles River Wistar (Crl:WI) rats $(n=6)$ weighing $180-220$ g were purchased from the Practical Skills and Experimental Medicine Centre of the "Iuliu Hațieganu" University of Medicine and Pharmacy (Cluj-Napoca, Romania). The animals were housed in polycarbonate type IV-S open-top cages (Tecniplast, Italy) at room temperature $22 \pm 2^{\circ} \mathrm{C}$, a relative humidity of $45 \% \pm 10 \%$, with a $12 / 12$ hours light/dark cycle. The animals received standard pelleted diet ("Cantacuzino" Institute, Bucharest, Romania) and water ad libitum. 24 hours before experiments, animals received only water in order to avoid food interference with substance absorption. All animal procedures were performed in accordance with the EEC Directive 63/2010, which regulates the care and use of laboratory animals for scientific purposes and were approved by The SanitaryVeterinary and Food Safety Directorate from Cluj County, Romania (66/06.06.2017). Animals were divided into groups $(n=6)$ and the synthesized compounds $(50 \mathrm{mg} / \mathrm{kg}$ bw) were solubilized in $0.5 \%$ Tween 80 solution and administered by gavage to 8 groups. The negative control group received only the vehicle ( $0.5 \%$ Tween 80 solution, by gavage) and the control positive group received diclofenac sodium (Gerot Lannach GmbH, Lannach, Austria) $(20 \mathrm{mg} / \mathrm{kg}$ bw, by gavage) as reference drug.

Anti-inflammatory activity. Anti-inflammatory activity was determined by $\lambda$-carrageenan-induced rat paw oedema test [42, 23]. Paw oedema was induced 1 hour after the intragastric administration of substances, by injecting $100 \mu \mathrm{L}$ of a $1 \% \lambda$-carrageenan (Sigma Aldrich, St. Louis, MO, USA) saline solution into left hind paw of the rat. The volume $(\mathrm{mL})$ of the paws was measured before and at 1, 2, 3 and 4 hours after $\lambda$-carrageenan injection. The volume of the oedema was measured using a digital plethysmometer (Ugo Basile 7140 Varese, Italy). Oedema volume and the percentage of oedema inhibition were expressed as follows:

$$
\text { Oedema volume }(\mathrm{mL})=\mathrm{Vt}-\mathrm{V} 0 \text {, }
$$

Inhibition of oedema $(\%)=[1-(\mathrm{Et} / \mathrm{Ec}) * 100]$, where, V0 is the mean paw volume before $\lambda$ carrageenan intraplantar injection, $\mathrm{Vt}$ is the mean paw volume at " $t$ " hours, Et is mean oedema volume in treated animals and Ec is mean oedema volume in the control group.

Antinociceptive activity. The nociceptive withdrawal threshold was assessed by Randall-Selitto test [9, 30]. For this experiment, to reduce the number of animals, the same groups of rats previously used for antiinflammatory testing were used. The negative control group (5\% Tween 80 solution), the positive control group (diclofenac sodium $20 \mathrm{mg} / \mathrm{kg} \mathrm{bw}$ ) and the other eight groups treated with synthesized compounds $(50 \mathrm{mg} / \mathrm{kg} \mathrm{bw})$ and receiving $100 \mu \mathrm{L} \lambda$-carrageenan $1 \%$ intraplantar injection 1 hour later, were tested for analgesia. The pain threshold of the inflamed hind paw of the rats was determined at 1, 2, 3 and 4 hours after the $\lambda$-carrageenan intraplantar injection, using an analgesimeter (Ugo Basile 37215, Varese, Italy). The analgesimeter was generating a linearly increased force (grams) until the animal produces a response characterized by removal of the paw, noises, interpreted as mechanical hypernociception. The instrument recorded the maximal amount of pressure (grams) withstood by rats at each time interval. The results are expressed as a percentage of the pressure applied and supported by the animals in inflammatory conditions, reported at time 0 which represents $100 \%$, prior to the induction of inflammation. We also highlighted the nociceptive threshold after the oral administration of synthesized compounds in this model of inflammatory pain, compared to diclofenac, as reference drug.

Ulcerogenic activity. Ulcerogenic activity of the synthesized compounds after a single oral administration of $50 \mathrm{mg} / \mathrm{kg}$ bw was investigated and scored by the method of Cioli et al. [8] adapted by Assarzadeh et al. [4]. All groups were fasted for 24 hours prior to drug administration and housed in mesh floor grid cages to avoid the coprophagy. Water was provided ad libitum. All animals were sacrificed under deep anaesthesia 6 hours after drug treatment then their stomachs were removed, opened along the great curvature and rinsed with saline solution $0.9 \%$. The gastric mucosa was examined by means of a magnifying 
glass (2X) to assess the incidence of redness and spot ulcers. The mucosal damage was evaluated according to the following score: 0.5 redness; 1.0 :spot ulcers; 1.5:haemorrhagic streaks; 2.0 ulcers $>3$ but $\leq 5$; and 3.0:ulcers $>5$. The gastric mucosal ulceration score was calculated by the difference between the mean score of each treated group and the mean score of control group.

Statistical data analysis

Statistics were performed with one-way analysis of variance (ANOVA), followed by Dunnett's multicomparison test. All data are presented as mean \pm standard error of mean (SEM), $\mathrm{p}<0.05$ was considered to be significant.

\section{Results and Discussion}

Chemistry

The general synthesis method of 4-(5-mercapto- $1 H$ 1,2,4-triazol-3-yl)benzenesulfonamide 4, is illustrated in Figure 1. The 4-sulfamoylbenzoic acid was converted into the corresponding methyl ester $\mathbf{1}$ and then treated with hydrazine hydrate in order to obtain 4-(hydrazinecarbonyl)benzenesulfonamide 2 . The 2-(4-sulfamoylbenzoyl)hydrazinecarbothioamide $\mathbf{3}$ previously obtained by the condensation of 4-(hydrazinecarbonyl)benzenesulfonamide 2 with potassium thiocyanate in acid catalysis, was converted to 4-(5-thioxo-4,5-dihydro- $1 \mathrm{H}$ 1,2,4-triazol-3-yl)benzenesulfonamide 4 by cyclization in basic conditions $[28,31]$. The Hantzsch condensation of the obtained 5-thioxotriazole 4 with $\alpha$-bromoketones lead to 4-(6-aryl-thiazolo[3,2-b][1,2,4]triazol2-yl)benzenesulfonamide derivatives 6a-6d, directly, when the condensation was in acid catalysis (Figure 2, Route A), or indirectly, by the cyclization of the corresponding 4-(5-((2-aryl-2-oxoethyl)thio)-1H1,2,4-triazol-3-yl)benzenesulfonamide 5a-5d (Figure 2, Route B) [10, 28, 44]. The 4-(6-aryl-thiazolo[3,2-b] $[1,2,4]$ triazol-2-yl)benzenesulfonamide derivatives 6a-6d and their corresponding thioethers 5a-5d were obtained in good yields $53 \%$ - 73\%, respectively $57 \%-88 \%$.

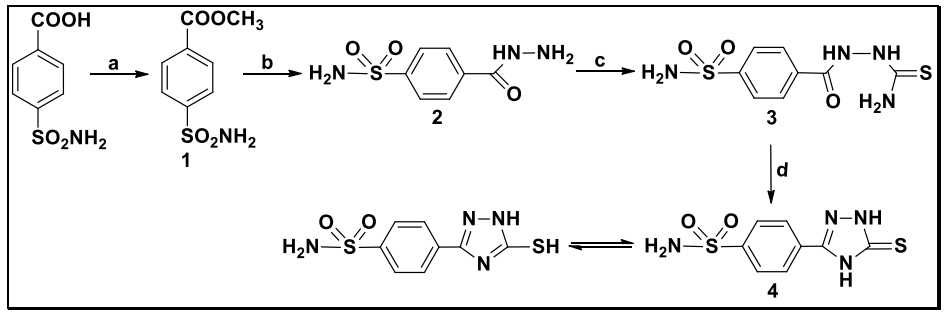

Figure 1.

The synthesis of 4-(5-thioxo-4,5-dihydro-1H-1,2,4-triazol-3-yl)benzenesulfonamide 4.

a. $\mathrm{CH}_{3} \mathrm{OH} / \mathrm{H}_{2} \mathrm{SO}_{4 \text { conc }}$, reflux; b. $\mathrm{NH}_{2}-\mathrm{NH}_{2}{ }^{*} \mathrm{H}_{2} \mathrm{O} / \mathrm{CH}_{3} \mathrm{OH}$, reflux; c. $\mathrm{KSCN} / \mathrm{HCl}_{\text {conc }}$, reflux; d. $\mathrm{NaOH} 10 \%$, reflux

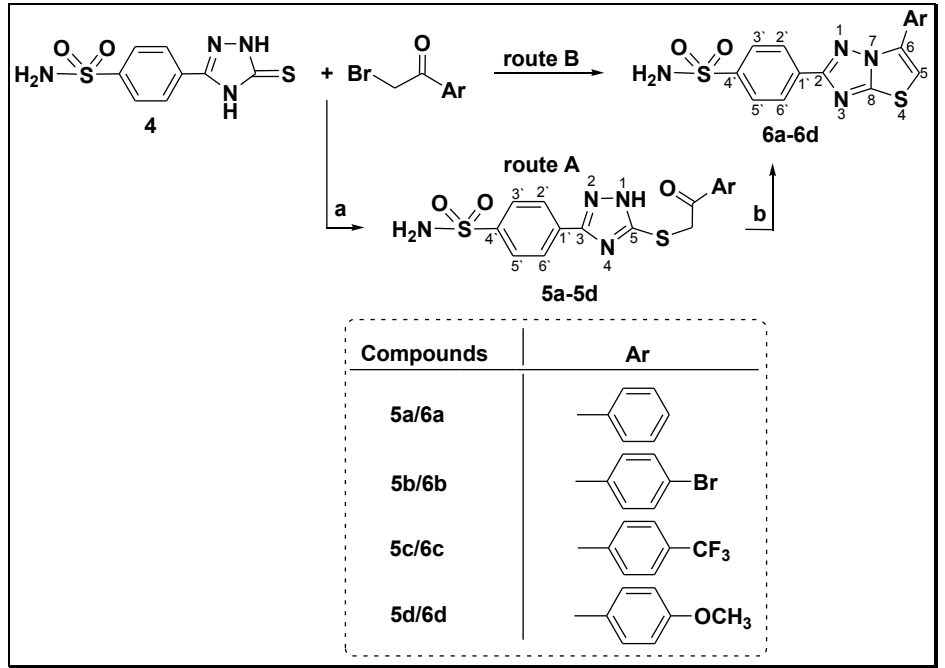

Figure 2.

The synthesis of thiazolo[3,2-b][1,2,4]triazole derivatives and the corresponding thioethers.

Route A: a. EtOH, $\mathrm{NaHCO}_{3}$, room temperature; b. $\mathrm{H}_{2} \mathrm{SO}_{4 \text { conc }}$. Route B: EtOH, $\mathrm{H}_{2} \mathrm{SO}_{4 \text { conc }}$, reflux

The synthesized compounds were purified and physicochemically characterized by melting points (m.p.), infrared spectra (IR), nuclear magnetic resonance ( ${ }^{1} \mathrm{H}$ NMR and $\left.{ }^{13} \mathrm{C} \mathrm{NMR}\right)$ and mass spectrometry
(MS). The details of the synthetic procedures of the synthesized compounds are presented in the Materials and Methods section. Some of the synthesized compounds $(\mathbf{5 a}, \mathbf{5 b}, \mathbf{5 d}, \mathbf{6 a}, \mathbf{6 b}, \mathbf{6 d})$ were reported in 
FARMACIA, 2018, Vol. 66, 5

the literature but their synthesis was achieved by a different method [18]. The yields of the synthesized compounds obtained by our method of synthesis were similar to those from the method reported previously. We improved the NMR spectral analysis of the compounds reported in the literature with the signals assignment of protons and carbon atoms. FT-IR spectra were recorded using the ATR technique and the results obtained were similar to those reported by Kumar et al. who used the $\mathrm{KBr}$ pellet technique [18]. 4-(5-((2-Oxo-2-phenylethyl)thio)-1H-1,2,4-triazol3-yl)benzenesulfonamide (5a)

Yield $57 \%(0.210 \mathrm{~g})$; purified by recrystallization from ethanol:water; m.p. $150-152^{\circ} \mathrm{C}\left(150-152^{\circ} \mathrm{C}[18]\right)$; yellow powder; FT-IR (ATR, $\mathrm{cm}^{-1}$ ): 3124, 2936, 2838 $(v \mathrm{~N}-\mathrm{H}, v \mathrm{C}-\mathrm{H}), 1609(\mathrm{vC}=\mathrm{O}) ;{ }^{1} \mathrm{H}-\mathrm{NMR}(600 \mathrm{MHz}$, DMSO- $\left.d_{6}, \delta / \mathrm{ppm}\right): 14.31$ (s, $\left.1 \mathrm{H}, \mathrm{NH}\right), 8.06(\mathrm{dd}, J=7.6$, $4.3 \mathrm{~Hz}, 4 \mathrm{H}, \mathrm{CH}-2$ ', CH-3', CH-5', CH-6'), 7.93 - 7.88 (m, 2H, CH-2, CH-6), 7.72 - 7.67 (m, 1H, CH-4), $7.58(\mathrm{t}, J=6.9 \mathrm{~Hz}, 2 \mathrm{H}, \mathrm{CH}-3, \mathrm{CH}-5), 7.51-7.42(\mathrm{~m}$, $\left.2 \mathrm{H}, \mathrm{NH}_{2}\right), 4.94$ (s, 2H, S-CH $) ;{ }^{13} \mathrm{C}-\mathrm{NMR}(151 \mathrm{MHz}$, DMSO- $\left.d_{6}, \delta / \mathrm{ppm}\right): 193.51(\mathrm{C}, \mathrm{C}=\mathrm{O}), 160.36(\mathrm{C}, \mathrm{C}-5$ 1,2,4-triazole), 154.05 (C, C-3 1,2,4-triazole), 152.14 (C, C-4'), 144.30 (C, C-1), 135.45 (CH, C-4), 133.77 (C, C-1'), 128.83 (CH, C-3', C-5'), 128.44 (CH, C-3, C-5), 126.48 (CH, C-2, C-6), 126.21 (CH, C-2', C-6'), $38.81\left(\mathrm{CH}_{2}, \mathrm{~S}-\mathrm{CH}_{2}\right)$; ESI ${ }^{+} \mathrm{MS}: m / z 375.5[\mathrm{M}+\mathrm{H}]^{+}$ (calculated: 375.4 for $\mathrm{C}_{16} \mathrm{H}_{14} \mathrm{~N}_{4} \mathrm{O}_{3} \mathrm{~S}_{2}[\mathrm{M}+\mathrm{H}]^{+}$). 4-(5-((2-(4-Bromophenyl)-2-oxoethyl)thio)-1H-1,2,4triazol-3-yl)benzenesulfonamide (5b)

Yield $88 \%$ ( $0.400 \mathrm{~g})$; purified by column chromatography, eluent dichloromethane: acetone 2:1 v/v; m.p. 194 $196^{\circ} \mathrm{C}\left(192-194^{\circ} \mathrm{C}[18]\right)$; yellow powder; FT-IR (ATR, $\left.\mathrm{cm}^{-1}\right)$ : 3252, $3100(v \mathrm{~N}-\mathrm{H}, v \mathrm{C}-\mathrm{H}), 1688(v \mathrm{C}=\mathrm{O}) ;{ }^{1} \mathrm{H}-$ NMR (600 MHz, DMSO- $\left.d_{6}, \delta / \mathrm{ppm}\right): 14.36$ (br s, $1 \mathrm{H}, \mathrm{NH}), 8.04$ (d, $J=8.4 \mathrm{~Hz}, 2 \mathrm{H}, \mathrm{CH}-3$ ', CH-5'), 7.99 (d, $J=8.6 \mathrm{~Hz}, 2 \mathrm{H}, \mathrm{CH}-2, \mathrm{CH}-6), 7.93-7.88$ (m, 2H, CH-3, CH-5), 7.80 (d, J=8.4 Hz, 2H, CH-2', CH-6'), 7.46 (s, 2H, NH $), 4.91\left(\mathrm{~s}, 2 \mathrm{H}, \mathrm{S}-\mathrm{CH}_{2}\right) ;{ }^{13} \mathrm{C}-$ NMR (151 MHz, DMSO- $\left.d_{6}, \delta / \mathrm{ppm}\right): 193.18(\mathrm{C}, \mathrm{C}=\mathrm{O})$, 160.27 (C, C-5 1,2,4-triazole), 159.37 (C, C-3 1,2,4triazole), 144.69 (C, C-4'), 134.74 (C, C-1), 134.66 (C, C-1'), 131.91 (CH, C-3, C-5), 130.45 (CH, C-3', C-5'), 130.23 (C, C-4), 127.80 (CH, C-2, C-6), 126.36 (CH, C-2', C-6'), $39.93\left(\mathrm{CH}_{2}, \mathrm{~S}^{-} \mathrm{CH}_{2}\right)$; ESI ${ }^{+}-\mathrm{MS}: \mathrm{m} / \mathrm{z}$ $455.2\left([\mathrm{M}+\mathrm{H}]^{+},{ }^{81} \mathrm{Br}\right), 453.7\left([\mathrm{M}+\mathrm{H}]^{+},{ }^{79} \mathrm{Br}\right)$ (calculated: $455.3{ }^{81} \mathrm{Br}, 453.9{ }^{79} \mathrm{Br}$ for $\left.\mathrm{C}_{16} \mathrm{H}_{13} \mathrm{BrN}_{4} \mathrm{O}_{3} \mathrm{~S}_{2}[\mathrm{M}+\mathrm{H}]^{+}\right)$. 4-(5-((2-Oxo-2-(4-(trifluoromethyl)phenyl)ethyl)thio)-1H-1,2,4-triazol-3-yl)benzenesulfonamide (5c) Yield $86 \%(0.380 \mathrm{~g})$; purified by column chromatography, eluent dichloromethane:acetone 2:1 v/v; m.p. 178 $180^{\circ} \mathrm{C}$; white powder; FT-IR (ATR, $\mathrm{cm}^{-1}$ ): 3262,3125 $(v \mathrm{~N}-\mathrm{H}, v \mathrm{C}-\mathrm{H}), 1693(v \mathrm{C}=\mathrm{O}) ;{ }^{1} \mathrm{H}-\mathrm{NMR}(600 \mathrm{MHz}$, DMSO- $\left.d_{6}, \delta / \mathrm{ppm}\right): 14.51$ (br s, $\left.1 \mathrm{H}, \mathrm{NH}\right), 8.25$ (d, $J=$ $8.0 \mathrm{~Hz}, 2 \mathrm{H}, \mathrm{CH}-2, \mathrm{CH}-6), 8.03(\mathrm{~d}, J=8.3 \mathrm{~Hz}, 2 \mathrm{H}$, CH-3', CH-5'), 7.96 (d, $J=8.1 \mathrm{~Hz}, 2 \mathrm{H}, \mathrm{CH}-3, \mathrm{CH}-5)$, 7.90 (d, $J=8.0 \mathrm{~Hz}, 2 \mathrm{H}, \mathrm{CH}-2$ ', CH-6'), 7.46 (s, 2H, $\left.\mathrm{NH}_{2}\right), 4.97\left(\mathrm{~s}, 2 \mathrm{H}, \mathrm{S}-\mathrm{CH}_{2}\right) ;{ }^{13} \mathrm{C}-\mathrm{NMR}(151 \mathrm{MHz}$,
DMSO- $\left.d_{6}, \delta / \mathrm{ppm}\right): 193.52(\mathrm{C}, \mathrm{C}=\mathrm{O}), 152.17(\mathrm{C}, \mathrm{C}-5$ 1,2,4-triazole), 144.86 (C, C-3 1,2,4-triazole), 143.20 (C, C-4'), 138.94 (C, C-1), 132.84 (q, J=31.3 Hz, C, C-4), 129.27 (C, C-1'), 126.36 (CH, C-3', C-5'), 126.27 (CH, C-2, C-6), 125.83 (CH, C-3, C-5), 125.81 (CH, C-2', C-6'), 123.78 (dd, $J=545.4,272.6 \mathrm{~Hz}, \mathrm{C}$, $\left.\mathrm{CF}_{3}\right), 40.06\left(\mathrm{CH}_{2}, \mathrm{~S}_{-} \mathrm{CH}_{2}\right)$; ESI ${ }^{+}-\mathrm{MS}: \mathrm{m} / z 443.1[\mathrm{M}+\mathrm{H}]^{+}$ (calculated: 443.4 for $\mathrm{C}_{17} \mathrm{H}_{13} \mathrm{~F}_{3} \mathrm{~N}_{4} \mathrm{O}_{3} \mathrm{~S}_{2}[\mathrm{M}+\mathrm{H}]^{+}$). 4-(5-((2-(4-Methoxyphenyl)-2-oxoethyl)thio)- $1 \mathrm{H}$ 1,2,4-triazol-3-yl)benzenesulfonamide (5d)

Yield $77 \%(0.310 \mathrm{~g})$; purified by column chromatography, eluent dichloromethane:acetone 2:1 v/v; m.p. 188 $190^{\circ} \mathrm{C}\left(214-216^{\circ} \mathrm{C}\right.$ [18]); white powder; FT-IR (ATR, $\left.\mathrm{cm}^{-1}\right): 3333,3260,2912(v \mathrm{~N}-\mathrm{H}, \mathrm{vC}-\mathrm{H}), 1589(\mathrm{vC}=\mathrm{O})$; ${ }^{1} \mathrm{H}-\mathrm{NMR}\left(600 \mathrm{MHz}, \mathrm{DMSO}-d_{6}, \delta / \mathrm{ppm}\right): 8.08(\mathrm{~d}, J=$ $8.4 \mathrm{~Hz}, 2 \mathrm{H}, \mathrm{CH}-3$ ', CH-5'), 8.04 (d, $J=8.8 \mathrm{~Hz}, 2 \mathrm{H}$, CH-2, CH-6), 7.91 (d, $J=8.4$ Hz, 2H, CH-2', CH-6'), 7.46 (s, 2H, NH$\left.)_{2}\right) 7.09$ (d, $\left.J=8.8 \mathrm{~Hz}, 2 \mathrm{H}, \mathrm{CH}-3, \mathrm{CH}-5\right)$, $4.88\left(\mathrm{~s}, 2 \mathrm{H}, \mathrm{S}-\mathrm{CH}_{2}\right), 3.86\left(\mathrm{~s}, 3 \mathrm{H}, \mathrm{O}-\mathrm{CH}_{3}\right) ;{ }^{13} \mathrm{C}-\mathrm{NMR}$ (151 MHz, DMSO- $\left.d_{6}, \delta / \mathrm{ppm}\right): 192.08(\mathrm{C}, \mathrm{C}=\mathrm{O}), 163.55$ (C, C-5 1,2,4-triazole), 157.63 (C, C-4), 155.46 (C, C-3 1,2,4-triazole), 144.70 (C, C-4'), 132.03 (C, C-1'), 130.86 (CH, C-3', C-5'), 128.39 (C, C-1), 126.34 (CH, C-2, C-6), 126.24 (CH, C-2', C-6'), 114.05 (CH, C-3, $\mathrm{C}-5), 55.67\left(\mathrm{CH}_{3}, \mathrm{O}-\mathrm{CH}_{3}\right), 39.93\left(\mathrm{CH}_{2}, \mathrm{~S}_{-} \mathrm{CH}_{2}\right)$; ESI $-\mathrm{MS}$ : $m / z 405.2[\mathrm{M}+\mathrm{H}]^{+}$(calculated: 405.4 for $\mathrm{C}_{17} \mathrm{H}_{16} \mathrm{~N}_{4} \mathrm{O}_{4} \mathrm{~S}_{2}$ $\left.[\mathrm{M}+\mathrm{H}]^{+}\right)$.

4-(6-Phenylthiazolo[3,2-b][1,2,4]triazol-2-yl)benzenesulfonamide (6a)

Yield 73\% (1.300 g); purified by column chromatography, eluent dichloromethane:acetone 2:1 v/v; m.p. 282 $284^{\circ} \mathrm{C}\left(284^{\circ} \mathrm{C}\right.$ [18]); yellow powder; FT-IR (ATR, $\left.\mathrm{cm}^{-1}\right)$ : 3308, $3128(\mathrm{vN}-\mathrm{H}, \mathrm{vC}-\mathrm{H})$; ${ }^{1} \mathrm{H}-\mathrm{NMR}(600 \mathrm{MHz}$, DMSO- $\left.d_{6}, \delta / \mathrm{ppm}\right): 8.32(\mathrm{~d}, J=8.5 \mathrm{~Hz}, 2 \mathrm{H}, \mathrm{CH}-3$ ', CH-5'), 8.28 (d, $J=7.3 \mathrm{~Hz}, 2 \mathrm{H}, \mathrm{CH}-2, \mathrm{CH}-6), 7.99$ (d, $J=8.5 \mathrm{~Hz}, 2 \mathrm{H}, \mathrm{CH}-2$ ', CH-6') overlapped with 7.98 (s, 1H, CH-5 thiazolo[3,2-b][1,2,4]triazole), 7.61 (t, $J=7.6 \mathrm{~Hz}, 2 \mathrm{H}, \mathrm{CH}-3, \mathrm{CH}-5), 7.54$ (t, $J=7.4 \mathrm{~Hz}$, $1 \mathrm{H}, \mathrm{CH}-4), 7.50$ (s, 2H, NH ); ${ }^{13} \mathrm{C}-\mathrm{NMR}(151 \mathrm{MHz}$, DMSO- $\left.d_{6}, \delta / \mathrm{ppm}\right): 164.47$ (C, C-8 thiazolo[3,2-b] $[1,2,4]$ triazole), 157.95 (C, C-2 thiazolo[3,2-b][1,2,4]triazole), 145.02 (C, C-4 ), 133.78 (C, C-6 thiazolo[3,2-b][1,2,4]triazole), $131.68\left(\mathrm{C}, \mathrm{C}-1^{\prime}\right), 129.78(\mathrm{CH}$, C-3', C-5'), 129.05 (C, C-1), 127.65 (CH, C-3, C-5), 126.74 (CH, C-4), 126.44 (CH, C-2, C-6), 126.42 (CH, C-2', C-6'), 111.36 (CH, C-5 thiazolo[3,2-b][1,2,4]triazole); $\mathrm{ESI}^{+}-\mathrm{MS}$ : $m / z 357.2[\mathrm{M}+\mathrm{H}]^{+}$(calculated: 357.4 for $\left.\mathrm{C}_{16} \mathrm{H}_{12} \mathrm{~N}_{4} \mathrm{O}_{2} \mathrm{~S}_{2}[\mathrm{M}+\mathrm{H}]^{+}\right)$.

4-(6-(4-Bromophenyl)thiazolo[3,2-b] [1,2,4]triazol2-yl)benzenesulfonamide (6b)

Yield 66\% (0.220 g); purified by column chromatography, eluent dichloromethane:acetone 2:1 v/v; m.p. 272 $274^{\circ} \mathrm{C}\left(270-272^{\circ} \mathrm{C}\right.$ [18]); yellow powder; FT-IR $\left(\mathrm{ATR}, \mathrm{cm}^{-1}\right): 3298,3126(v \mathrm{~N}-\mathrm{H}, v \mathrm{C}-\mathrm{H}) ;{ }^{1} \mathrm{H}-\mathrm{NMR}$ (600 MHz, DMSO- $\left.d_{6},{ }_{\delta} / \mathrm{ppm}\right): 8.31(\mathrm{~d}, J=8.4 \mathrm{~Hz}$, 2H, CH-3', CH-5'), 8.25 (d, $J=8.6 \mathrm{~Hz}, 2 \mathrm{H}, \mathrm{CH}-2$, $\mathrm{CH}-6), 8.07$ (s, 1H, CH-5 thiazolo[3,2-b][1,2,4]triazole), 7.98 (d, $J=8.4 \mathrm{~Hz}, 2 \mathrm{H}, \mathrm{CH}-2$ ', CH-6'), 7.81 
FARMACIA, 2018, Vol. 66, 5

(d, $J=8.6 \mathrm{~Hz}, 2 \mathrm{H}, \mathrm{CH}-3, \mathrm{CH}-5), 7.50$ (s, 2H, NH ); ${ }^{13} \mathrm{C}-\mathrm{NMR}\left(151 \mathrm{MHz}, \mathrm{DMSO}-d_{6}, \delta / \mathrm{ppm}\right) 164.49$ (C, C-8 thiazolo[3,2-b][1,2,4]triazole), 157.97 (C, C-2 thiazolo[3,2-b][1,2,4]triazole), 145.07 (C, C-4) 133.68 (C, C-6 thiazolo[3,2-b][1,2,4]triazole), $132.03(\mathrm{CH}$, C-3, C-5), 130.59 ( C, C-1'), 128.31 (CH, C-3`, C-5'), 126.82 (C, C-1), 126.73 (CH, C-2, C-6), $126.42(\mathrm{CH}$, C-2', C-6'), 123.05 (C, C-4), 112.17 (C, C-5 thiazolo[3,2-b][1,2,4]triazole); ESI ${ }^{+}-\mathrm{MS}: m / z 435.8\left([\mathrm{M}+\mathrm{H}]^{+}\right.$, $\left.{ }^{79} \mathrm{Br}\right), 437.2\left([\mathrm{M}+\mathrm{H}]{ }^{+},{ }^{81} \mathrm{Br}\right)$ (calculated: $435.9{ }^{79} \mathrm{Br}$, $437.3{ }^{81} \mathrm{Br}$ for $\left.\mathrm{C}_{16} \mathrm{H}_{13} \mathrm{BrN}_{4} \mathrm{O}_{3} \mathrm{~S}_{2}[\mathrm{M}+\mathrm{H}]^{+}\right)$.

4-(6-(4-(Trifluoromethyl)phenyl)thiazolo[3,2-b]

[1,2,4] triazol-2-yl)benzenesulfonamide (6c)

Yield $62 \%$ ( $0.050 \mathrm{~g})$; purified by column chromatography, eluent dichloromethane:acetone 2:1 v/v; m.p. 234 $236^{\circ} \mathrm{C}$; yellow powder; FT-IR (ATR, $\mathrm{cm}^{-1}$ ): 3276 , 3150 (vN-H, vC-H); ${ }^{1} \mathrm{H}-\mathrm{NMR}\left(600 \mathrm{MHz}, \mathrm{DMSO}-d_{6}\right.$, $\delta / \mathrm{ppm}): 8.50(\mathrm{~d}, J=8.2 \mathrm{~Hz}, 2 \mathrm{H}, \mathrm{CH}-3$ ', CH-5'), 8.32 (d, $J=8.4 \mathrm{~Hz}, 2 \mathrm{H}, \mathrm{CH}-5, \mathrm{CH}-5), 8.19$ (s, 1H, CH-5 thiazolo[3,2-b][1,2,4]triazole), 7.99 (d, $J=8.4 \mathrm{~Hz}, 2 \mathrm{H}$, $\mathrm{CH}-2, \mathrm{CH}-6), 7.96$ (d, $\left.J=8.3 \mathrm{~Hz}, 2 \mathrm{H}, \mathrm{CH}-2{ }^{\prime}, \mathrm{CH}-6 '\right)$, $7.50\left(\mathrm{~s}, 2 \mathrm{H}, \mathrm{NH}_{2}\right) ;{ }^{13} \mathrm{C}-\mathrm{NMR}\left(151 \mathrm{MHz}, \mathrm{DMSO}-d_{6}\right.$, $\delta / \mathrm{ppm}): 164.52$ (C, C-8 thiazolo[3,2-b][1,2,4]triazole) 158.01 (C, C-2 thiazolo[3,2-b][1,2,4]triazole), 145.11 (C, C-4'), 133.65 (C, C-6 thiazolo[3,2-b][1,2,4]triazole), 131.36 (C, C-1), 130.21 (C, C-1'), 129.50 (q, $\mathrm{J}=32.3 \mathrm{~Hz}, \mathrm{C}-4), 127.03$ (C, C-3', C-5'), 126.74 (C, C-2, C-6 ), 126.42 (C, C-3, C-5), 125.94 (C, C-2', C-6'), 126.9-120.95 (m, $\left.\mathrm{CF}_{3}\right), 114.06$ (C, C-5 thiazolo[3,2-b][1,2,4]triazole); ESI ${ }^{+}-\mathrm{MS}: m / z 425.0[\mathrm{M}+\mathrm{H}]^{+}$ (calculated: 425.4 for $\mathrm{C}_{17} \mathrm{H}_{11} \mathrm{~F}_{3} \mathrm{~N}_{4} \mathrm{O}_{2} \mathrm{~S}_{2}[\mathrm{M}+\mathrm{H}]^{+}$). 4-(6-(4-Methoxyphenyl)thiazolo[3,2-b][1,2,4]triazol-2-yl)benzenesulfonamide (6d)

Yield 53\% (0.150 g); purified by recrystallization from ethanol:water; m.p. $254-256^{\circ} \mathrm{C}\left(256^{\circ} \mathrm{C}[18]\right)$; yellow powder; FT-IR (ATR, $\left.\mathrm{cm}^{-1}\right): 3257,3125(\mathrm{vN}-\mathrm{H}$, vC-H); ${ }^{1} \mathrm{H}-\mathrm{NMR}\left(600 \mathrm{MHz}, \mathrm{DMSO}-d_{6}, \delta / \mathrm{ppm}\right): 8.31$ (d, $\left.J=8.4 \mathrm{~Hz}, 2 \mathrm{H}, \mathrm{CH}-3^{\prime}, \mathrm{CH}-5^{\prime}\right), 8.23$ (d, $J=8.8 \mathrm{~Hz}$, 2H, CH-2, CH-6), 7.98 (d, $J=8.4 \mathrm{~Hz}, 2 \mathrm{H}, \mathrm{CH}-2$ ', CH-6'), 7.83 (s, 1H, CH-5 thiazolo[3,2-b][1,2,4]triazole), 7.49 (s, 2H, $\left.\mathrm{NH}_{2}\right), 7.16(\mathrm{~d}, J=8.9 \mathrm{~Hz}, 2 \mathrm{H}, \mathrm{CH}-3$, $\mathrm{CH}-5), 3.85$ (s, 3H, O-CH 3 ); ${ }^{13} \mathrm{C}-\mathrm{NMR}(151 \mathrm{MHz}$, DMSO- $\left.d_{6}, \delta / \mathrm{ppm}\right): 164.41$ (C, C-8 thiazolo[3,2-b] $[1,2,4]$ triazole), 160.29 (C, C-4), 157.86 (C, C-2 thiazolo[3,2-b][1,2,4]triazole), 144.99 (C, C-4) 133.83 (C, C-6 thiazolo[3,2-b][1,2,4]triazole), 131.57 (C, C-1 '), 128.01 (CH, C-3`, C-5'), 126.70 (CH, C-2, C-6), 126.44 (CH, C-2', C-6'), 120.16 (C, C-1), 114.44 (CH, C-3, C-5), 109.06 (C, C-5 thiazolo[3,2-b][1,2,4]triazole), $55.40\left(\mathrm{CH}_{3}, \mathrm{O}-\mathrm{CH}_{3}\right)$; $\mathrm{ESI}^{+}-\mathrm{MS}: \mathrm{m} / z 387.2[\mathrm{M}+\mathrm{H}]^{+}$ (calculated: 387.4 for $\mathrm{C}_{17} \mathrm{H}_{14} \mathrm{~N}_{4} \mathrm{O}_{3} \mathrm{~S}_{2}[\mathrm{M}+\mathrm{H}]^{+}$).

In the IR spectra of the 4-(5-((2-aryl-2-oxoethyl)thio)-1H-1,2,4-triazol-3-yl)benzenesulfonamide 5a5d, the carbonyl stretching bands $v \mathrm{C}=\mathrm{O}$ appeared at $1693-1589 \mathrm{~cm}^{-1}$, while in the IR spectra of the corresponding 4-(6-aryl-thiazolo[3,2-b][1,2,4]triazol-2-yl)benzenesulfonamides 6a-6d, the carbonyl stretching band is missing. This fact confirms the ring closure of the acyclic compounds 5a-5d due to cyclization reaction. The presence of sulfonamide group in the IR spectra of all synthesized compounds was indicated by $-\mathrm{NH}_{2}$ stretching vibrational band.

The ${ }^{1} \mathrm{H}$ NMR spectra of compounds 5a-5d displayed a single signal at $\delta 14.31-14.51 \mathrm{ppm}$, corresponding to $\mathrm{NH}$ proton from $1 \mathrm{H}-1,2,4-$ triazole ring. The presence of a singlet at $\delta 4.88-4.97$ ppm corresponding to the protons of the thiomethylene group $-\mathrm{S}-\mathrm{CH}_{2}$ - indicates the formation of alkylated triazoles $\mathbf{5 a - 5 d}$. In the ${ }^{1} \mathrm{H}$ NMR spectra of the 6a-6d compounds, the signals corresponding to the protons from $\mathrm{NH}$ and thiomethylene group are not present, thus confirming that the cyclisation of the compounds 5a-5d occurred. Also, the presence of a singlet in the aromatic area at $\delta 7.83-8.19 \mathrm{ppm}$ corresponding to the proton from the $5^{\text {th }}$ position of the thiazolo[3,2-b][1,2,4]triazole ring indicates the formation of the fused ring system. For all compounds, the signals corresponding to the protons from $\mathrm{NH}_{2}$ group are present as a singlet at $\delta$ $7.46-7.50 \mathrm{ppm}$.

The ${ }^{13} \mathrm{C}$ NMR spectra of 4-(5-((2-aryl-2-oxoethyl)thio)-1 $H$-1,2,4-triazol-3-yl)benzenesulfonamide 5a5d revealed the presence of the aliphatic signals corresponding to the carbon atoms of the thiomethylene group (-S- $\left.\mathrm{CH}_{2}-\right)$ at $\delta 38.81-40.06 \mathrm{ppm}$, as well as the characteristic carbonyl signals $(>\mathrm{C}=\mathrm{O})$ at $\delta 192.08$ - $193.52 \mathrm{ppm}$. These signals are not present in the ${ }^{13} \mathrm{C}$ NMR spectra of the cyclization products 6a-6d, this fact representing another proof that the cyclization of the compounds 5a-5d was achieved. In the ${ }^{13} \mathrm{C}$ NMR spectra of thiazolo[3,2-b][1,2,4]triazole derivatives $\mathbf{6 a - 6 d}$ the aliphatic signals of carbon atoms from thiomethylene group $\left(-\mathrm{S}_{-}-\mathrm{CH}_{2}-\right)$ and carbonyl $(>\mathrm{C}=\mathrm{O})$ signals are replaced by two aromatic signals corresponding to the carbon atoms from the $5^{\text {th }}$ and $6^{\text {th }}$ positions of the thiazolo[3,2-b][1,2,4]triazole ring system. In the ${ }^{13} \mathrm{C}$ NMR spectra of trifluoromethyl derivative $\mathbf{5 c}$ and its corresponding cyclized compound $\mathbf{6 c}$, it was observed the presence of a characteristic quartet for $\mathrm{CF}_{3}$ group. The $\mathrm{CF}_{3}$ carbon signal was split into a quartet of intensities $1: 3: 3: 1$ due to the effect of the three fluorine nuclei. The coupling constant $J$ of the $\mathrm{CF}_{3}$ quartet is approximately $272 \mathrm{~Hz}$.

All signals of the carbon atoms of the aromatic rings are present in the aromatic region and the aliphatic signals corresponding to the carbon atoms from the methyl groups are also present.

The MS spectra confirmed the structures of the synthesized compounds 5a-5d, 6a-6d by revealing the presence of the molecular ion $[\mathrm{M}+\mathrm{H}]^{+}$peak in different intensities. The MS spectra of the compounds 5b and $\mathbf{6 b}$ with bromine atoms in their structure, presented characteristic peaks corresponding to the two isotopes $\left({ }^{79} \mathrm{Br}\right.$ and $\left.{ }^{81} \mathrm{Br}\right)$ and their intensity was in accordance with the relative distribution of the two isotopes. 
FARMACIA, 2018, Vol. 66, 5

MS spectra confirmed the structures of all synthesized compounds by the presence of the molecular peak. Anti-inflammatory activity

All the synthesized compounds and diclofenac, as reference drug, were tested for their in vivo anti- inflammatory activity by modified carrageenan-induced rat paw oedema model, firstly described by Winter et al. [42]. The results of anti-inflammatory activities against carrageenan-induced paw oedema are shown in Table I.

Table I

In vivo anti-inflammatory activity of synthesized compounds on carrageenan-induced paw oedema in rats

\begin{tabular}{|c|c|c|c|c|c|}
\hline Compound & $\begin{array}{c}\begin{array}{c}\text { Dose } \\
\text { (mg/kg) p.o. }\end{array} \\
\end{array}$ & $\begin{array}{c}\text { Oedema } 1 \text { h }(\mathrm{mL}) \\
\text { (\%inhib.) }\end{array}$ & $\begin{array}{c}\text { Oedema } 2 \text { h }(\mathrm{mL}) \\
\text { (\%inhib.) }\end{array}$ & $\begin{array}{c}\text { Oedema } 3 \mathrm{~h}(\mathrm{~mL}) \\
\text { (\%inhib.) }\end{array}$ & $\begin{array}{c}\text { Oedema } 4 \text { h }(\mathrm{mL}) \\
(\% \text { inhib.) }\end{array}$ \\
\hline $\begin{array}{l}\text { Negative control } \\
\text { (vehicle) }\end{array}$ & - & $0.80 \pm 0.10$ & $1.66 \pm 0.12$ & $2.43 \pm 0.10$ & $1.63 \pm 0.17$ \\
\hline \multirow[t]{2}{*}{$5 a$} & 50 & $0.74 \pm 0.20$ & $1.62 \pm 0.20$ & $2.40 \pm 0.28$ & $1.48 \pm 0.29$ \\
\hline & & $(6.69)$ & $(2.12)$ & $(1.30)$ & $(9.09)$ \\
\hline \multirow[t]{2}{*}{$5 \mathbf{b}$} & 50 & $0.70 \pm 0.14$ & $1.23 \pm 0.17$ & $1.80 \pm 0.26$ & $0.79 \pm 0.19 *$ \\
\hline & & $(11.72)$ & $(25.60)$ & $(26.15)$ & $(51.38)$ \\
\hline \multirow[t]{2}{*}{$5 c$} & 50 & $0.70 \pm 0.18$ & $1.04 \pm 0.10$ & $1.54 \pm 0.15^{*}$ & $1.17 \pm 0.24$ \\
\hline & & (12.34) & $(37.00)$ & $(36.76)$ & $(28.40)$ \\
\hline \multirow[t]{2}{*}{$5 d$} & 50 & $0.77 \pm 0.13$ & $1.58 \pm 0.20$ & $2.03 \pm 0.29$ & $0.92 \pm 0.27$ \\
\hline & & $(3.56)$ & $(4.33)$ & $(16.63)$ & $(43.31)$ \\
\hline \multirow[t]{2}{*}{$6 a$} & 50 & $0.65 \pm 0.09$ & $1.38 \pm 0.08$ & $2.08 \pm 0.18$ & $0.84 \pm 0.21$ \\
\hline & & (18.41) & $(16.43)$ & $(14.72)$ & (48.31) \\
\hline \multirow[t]{2}{*}{$6 \mathrm{~b}$} & 50 & $0.79 \pm 0.10$ & $1.44 \pm 0.16$ & $2.12 \pm 0.20$ & $1.18 \pm 0.24$ \\
\hline & & $(0.42)$ & $(12.80)$ & $(12.87)$ & $(27.68)$ \\
\hline \multirow[t]{2}{*}{$6 c$} & 50 & $0.65 \pm 0.12$ & $1.47 \pm 0.20$ & $2.23 \pm 0.24$ & $1.36 \pm 0.17$ \\
\hline & & $(18.43)$ & $(10.79)$ & $(8.35)$ & $(16.65)$ \\
\hline \multirow[t]{2}{*}{ 6d } & 50 & $0.73 \pm 0.16$ & $0.92 \pm 0.16 *$ & $1.80 \pm 0.16$ & $1.13 \pm 0.15$ \\
\hline & & $(7.95)$ & $(44.15)$ & $(26.01)$ & $(30.85)$ \\
\hline \multirow{2}{*}{$\begin{array}{c}\text { Diclofenac } \\
\text { (reference drug) }\end{array}$} & 20 & $0.67 \pm 0.10$ & $0.68 \pm 0.12 *$ & $1.15 \pm 0.16^{*}$ & $0.66 \pm 0.24^{*}$ \\
\hline & & $(16.11)$ & $(58.97)$ & $(52.84)$ & $(59.24)$ \\
\hline
\end{tabular}

All synthesized compounds 5a-5d, 6a-6d reduced carrageenan-induced paw oedema when administered in dose of $50 \mathrm{mg} / \mathrm{kg}$ bw, p.o., compared to negative control group. Diclofenac $(20 \mathrm{mg} / \mathrm{kg}$ bw, p.o.), a nonselective NSAID used as reference drug, significantly reduced paw volumes at all periods of time, compared to negative control group. When compared to diclofenac as reference drug, the synthesized compounds showed moderate to good anti-inflammatory activity, but the results were not statistically significant. After 1 hour from the inflammation induction, compounds $\mathbf{6 a}$ (18.41\%) and 6c (18.43\%), showed an anti-inflammatory activity superior to diclofenac (16.11\%). At 2 hours after $\lambda$-carrageenan injection, the compounds $\mathbf{5 c}$ $(37.00 \%)$ and $6 \mathbf{d}(44.15 \%)$ produced an important inhibition of oedema compared to diclofenac (58.97\%). Compounds $\mathbf{5 c}(36.76 \%)$ and $\mathbf{5 b}(51.38 \%)$ significantly decreased paw oedema at 3 , respectively 4 hours after the induction of inflammation. At 4 hours after the inflammation occurred, compounds $\mathbf{5 d}$ (43.31\%), 6a $(48.31 \%)$, 6d $(30.85 \%)$ possessed good antiinflammatory activity compared to diclofenac (59.24\%). The peripheral inflammatory response to carrageenan is characterized by the cardinal signs of inflammation and has a biphasic mechanism [40]. The initial oedematous phase $(0-1 \mathrm{~h})$ is mediated by histamine and 5-hydroxytriptamine, followed by release of bradykinin. The second phase $(1-4 \mathrm{~h})$ is attributed to an increase of prostaglandins in the damaged tissue $[11,25,41]$. The mechanism of $\lambda$-carrageenan`s effects in inflammation is explained by the interaction of $\lambda$-carrageenan with Toll-like receptor 4 which induces the activation of Nuclear Factor $\kappa \mathrm{B}(\mathrm{NF} \kappa \mathrm{B})$ and interleukin 8 (IL-8) through a pathway of innate immunity mediated by B-cell CLL/lymphoma 10 [6]. $\mathrm{NF}-\kappa \mathrm{B}$ is known to be a major transcription factor for regulating the expressions of proinflammatory enzymes and cytokines, such as iNOS, COX-2, and TNF- $\alpha$ [16]. COX-2 mediates the formation of prostaglandin E2 and prostaglandin I2 which causes increase of vasodilatation, vascular permeability and extravasation of plasma proteins. The mechanism of action of NSAIDs is correlated with the inhibition of COX-1 and COX-2 isoenzymes [31]. Compound $6 \mathbf{6 a}$ with phenyl moiety and $\mathbf{6 c}$ with trifluoromethyl moiety showed a better anti-inflammatory activity on the initial oedematous phase than diclofenac as reference drug. Compound 6a maintained its moderate to good anti-inflammatory activity at 2, 3, 4 hours after the inflammation induction, along with the compounds substituted with methoxy $\mathbf{5 d}$ and $\mathbf{6 d}$, bromide $\mathbf{5 b}$, and trifluoromethyl 5c moieties. The structure-activity relationship studies suggests that the presence of unsubstituted phenyl inhibit both COX-1 and COX-2 
FARMACIA, 2018, Vol. 66, 5

and the presence of $\mathrm{OCH}_{3}$ and $\mathrm{F}$ groups at the $4^{\text {th }}$ position of the 6-phenyl ring contributes for selective COX-2 inhibitory activity [14]. The good antiinflammatory activity of compounds $\mathbf{5 d}, \mathbf{6 d}$, could be assumed also to a possible hydrogen bound formation between the oxygens of $\mathrm{OCH}_{3}$ groups with the binding site of the $\mathrm{COX}$ [4]. The compounds bearing bromide 5b and trifluoromethyl 5c substituents, showed noticeable anti-inflammatory and analgesic activities and in accordance with the literature data, proves the importance of the halogens presence for the antiinflammatory activity [12].

\section{Antinociceptive activity}

The antinociceptive activity of the synthesized compounds in a model of inflammatory pain was evaluated by Randall-Selitto test. The oral administration of the synthesized compounds $\mathbf{5 a - 5 d}, \mathbf{6 a - 6} \mathbf{d}$ in doses of $50 \mathrm{mg} / \mathrm{kg}$ bw, produced a good increase of nociceptive threshold when compared to negative control group. Some compounds showed a significant increase of nociceptive threshold when compared to diclofenac, as reference drug. The results of Randall-Selitto test are shown in Figure 3 and Table II.

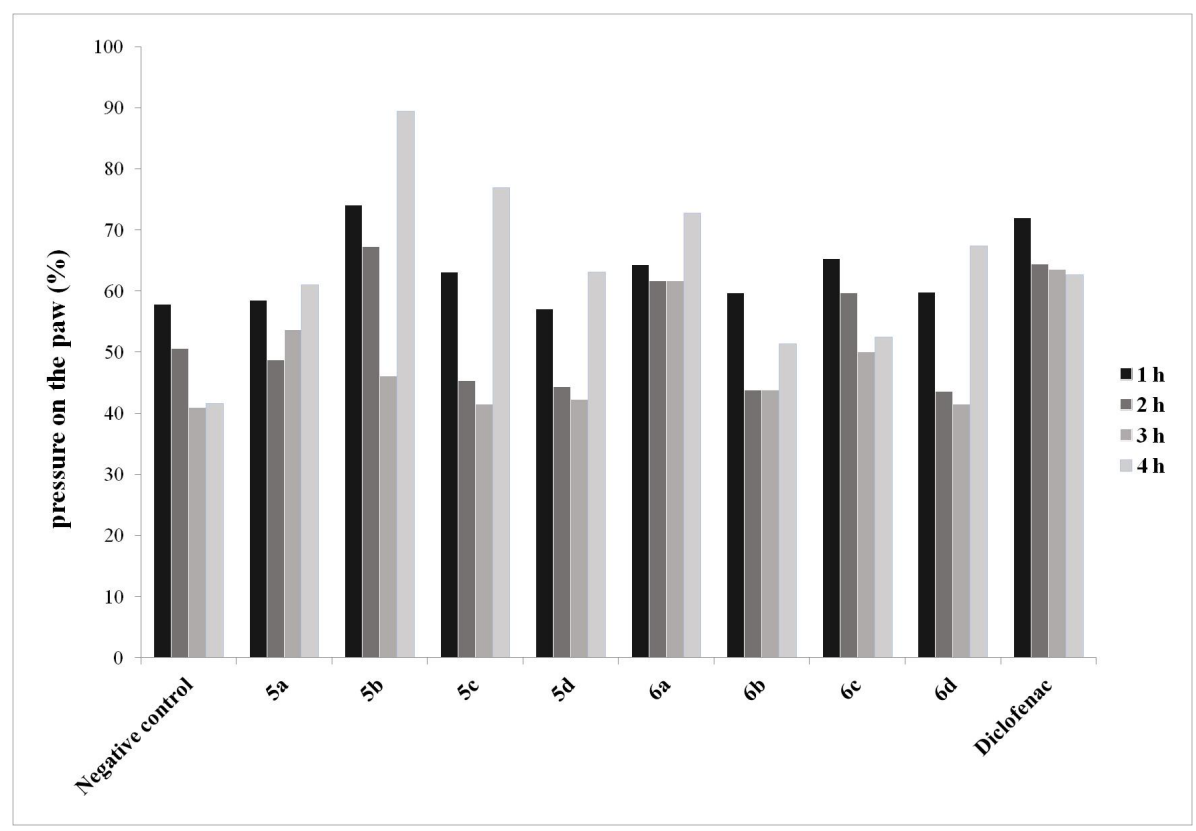

Figure 3.

Antinociceptive activity of synthesized compounds on paw pressure test in rats (Randall-Selitto test)

Table II

Nociceptive threshold after the oral administration of tested compounds (Randall-Selitto test)

\begin{tabular}{cccccc}
\hline Group & $\begin{array}{c}\text { Dose } \\
\text { (mg/kg) p.o. }\end{array}$ & \multicolumn{4}{c}{ Paw pressure (g) } \\
& - & $74.17 \pm 5.38$ & $65.00 \pm 3.41$ & $52.50 \pm 4.03$ & $53.33 \pm 4.41$ \\
\hline Negative control (vehicle) & 50 & $80.00 \pm 6.95$ & $66.67 \pm 8.62$ & $73.33 \pm 2.10$ & $\mathbf{8 3 . 3 3} \pm \mathbf{8 . 3 3 *}$ \\
$\mathbf{5 a}$ & 50 & $64.17 \pm 3.00$ & $58.33 \pm 5.27$ & $40.00 \pm 4.83$ & $\mathbf{7 7 . 5 0} \pm \mathbf{6 . 2 9 *}$ \\
$\mathbf{5 b}$ & 50 & $68.33 \pm 5.72$ & $49.17 \pm 3.27$ & $45.00 \pm 4.08$ & $\mathbf{8 3 . 3 3} \pm \mathbf{7 . 0 3 *}$ \\
$\mathbf{5 c}$ & 50 & $70.83 \pm 7.68$ & $55.00 \pm 3.87$ & $52.50 \pm 4.23$ & $78.33 \pm 8.81$ \\
$\mathbf{5 d}$ & 50 & $82.50 \pm 7.27$ & $79.17 \pm 6.24$ & $\mathbf{7 9 . 1 7} \pm \mathbf{8 . 4 0}$ & $\mathbf{9 3 . 3 3} \pm \mathbf{6 . 2 8 *}$ \\
6a & 50 & $71.67 \pm 4.21$ & $52.50 \pm 4.23$ & $52.50 \pm 3.09$ & $61.67 \pm 5.86$ \\
6b & 50 & $67.50 \pm 4.95$ & $61.67 \pm 4.21$ & $51.67 \pm 2.78$ & $54.17 \pm 3.96$ \\
6c & 50 & $73.33 \pm 7.14$ & $53.33 \pm 5.72$ & $50.83 \pm 3.00$ & $\mathbf{8 2 . 5 0} \pm \mathbf{5 . 8 8 *}$ \\
6d & 20 & $70.83 \pm 2.38$ & $63.33 \pm 4.77$ & $62.50 \pm 3.35$ & $61.67 \pm 3.80$ \\
\hline
\end{tabular}

* Statistically significant, $\mathrm{p}<0.05$. Values are expressed as mean \pm SEM.

In the negative control group, $\lambda$-carrageenan injected into the left hind paw induced a decrease in the nociceptive threshold of the left paw, in the same time with the development of oedema, from 1 to 4 hours. Compounds $5 \mathbf{a}$ and $\mathbf{6 a}$ showed a good increase in the nociceptive threshold, but the increase was significant only at 3 hours $(79.17 \pm 8.40,61.69 \%)$ and 4 hours
$(93.33 \pm 6.28,72.73 \%)$ after the inflammation induction for $\mathbf{6 a}$ and at 4 hours $(83.33 \pm 8.33,60.98 \%)$ for $\mathbf{5 a}$. Compounds 5b $(77.50 \pm 6.29,98.42 \%), \mathbf{5 c}(83.33 \pm$ $7.03,76.92 \%)$ and $\mathbf{6 d}(82.50 \pm 5.88,67.35 \%)$ presented a significant increase in nociception threshold at 4 hours when compared to diclofenac $(61.67 \pm 3.80$, $62.71 \%)$. 
The antinociceptive activity evaluated in a model of inflammatory pain showed that the decrease in threshold values was prevented in rats pre-treated with the synthesized compounds and then injected with carrageenan when compared to negative control group. This fact could be explained by the existence of the sulfonamide group, which is reported in the literature as a pharmacophore for hypoalgesia [15]. A significant raised nociceptive threshold in the inflamed paw was also observed for compounds with unsubstituted phenyl 5a, 6a and with phenyl substituted with bromide $\mathbf{5 b}$, trifluoromethyl $\mathbf{5 c}, \mathbf{6 c}$, and methoxy $\mathbf{6 d}$ moieties. Analgesic activity studies revealed that compounds with unsubstituted phenyl and with 4-bromophenyl and 4-fluorophenyl exhibited good analgesic activities $[4,12,17]$. The antinociceptive activity of the tested compounds was observed especially in the $3^{\text {rd }}$ and $4^{\text {th }}$ hours after the intra-plantar injection of carrageenan, which may suggest that the synthesized compounds influence the second phase of acute inflammation and that the anti-inflammatory effect appears over time.

Ulcerogenic activity

The synthesized compounds were evaluated for their ulcerogenic activity after a single oral administration of $50 \mathrm{mg} / \mathrm{kg}$ bw in rats. The gastric mucosa of rats was examined for irritations, ulcerations and microhaemorrhages by a magnifier. The evaluation of gastric mucosal lesions was according to their number and size, in a scale from 0.5 to 3.0 points, as detailed in Materials and Methods section. The results of ulcerogenic activity of the synthesized compounds are shown in Table III.

Table III

Ulcerogenic activity screening of the synthesized compounds

\begin{tabular}{ccc}
\hline Group & Dose (mg/kg) p.o. & Ulceration score \\
\hline Negative control (vehicle) & - & $0.00 \pm 0.00$ \\
$\mathbf{5 a}$ & 50 & $\mathbf{0 . 9 1} \pm \mathbf{0 . 0 8 *}$ \\
$\mathbf{5 b}$ & 50 & $\mathbf{0 . 5 8} \pm \mathbf{0 . 2 0}$ \\
$\mathbf{5 c}$ & 50 & $0.16 \pm 0.10$ \\
$\mathbf{5 d}$ & 50 & $\mathbf{0 . 7 5} \pm \mathbf{0 . 1 7}$ \\
$\mathbf{6 a}$ & 50 & $0.41 \pm 0.20$ \\
$\mathbf{6 b}$ & 50 & $0.50 \pm 0.12$ \\
$\mathbf{6 c}$ & 50 & $0.08 \pm 0.08$ \\
$\mathbf{6 d}$ & 50 & $0.41 \pm 0.15$ \\
Diclofenac (reference drug) & 20 & $\mathbf{2 . 6 6} \pm \mathbf{0 . 2 1}$ \\
\hline
\end{tabular}

*Statistically significant, $\mathrm{p}<0.05$. Values are expressed as mean \pm SEM.

The results were compared with those from negative control group, which were given only vehicle $(5 \%$ Tween 80 solution) and with diclofenac group. A significant reduction in ulcerogenic activity with the stomach mucosal ulceration score between $0.08 \pm 0.08$ and $0.50 \pm 0.12$ was observed for the tested compounds. Three compounds $5 \mathbf{5}(0.91 \pm 0.08), \mathbf{5 b}(0.58 \pm 0.20)$, 5d $(0.75 \pm 0.17)$ induced significant ulceration when compared to negative control group. However, none of the synthesized compounds presented significant ulceration when compared to diclofenac group.

Most of the classical NSAIDs are associated with side effects of the gastric mucosa at the dosage exerting their anti-inflammatory, analgesic and antipyretic activities. As we expected, diclofenac as a nonselective NSAID used as reference drug, when administered orally ( $20 \mathrm{mg} / \mathrm{kg} \mathrm{bw}$ ) showed significant ulcerogenic risk, with the highest ulceration score among all the synthesized compounds. Three compounds $\mathbf{5 a}, \mathbf{5 b}, \mathbf{5 d}$ of the 4-(5-((2-aryl-2-oxoethyl)thio)- $1 \mathrm{H}$ 1,2,4-triazol-3-yl)benzenesulfonamide derivatives presented significant stomach ulceration score when compared to negative control group, but the ulceration risk of all tested compounds was significantly reduced when compared to diclofenac group. This fact could be explained due to the presence of sulfonamide group which exhibit antiulcer potential by increasing
COX-2 selectivity and by inhibition of carbonic anhydrase activity, which may limit the acid secretion $[7,37]$. Some of the compounds with lower ulcerogenic risk $(\mathbf{5 b}, \mathbf{5 c}, 5 \mathbf{d}, \mathbf{6 a}, \mathbf{6 c}$ and $\mathbf{6 d})$ presented good antiinflammatory and analgesic effects after oral administration.

The molecular mechanism of action of the compounds with good anti-inflammatory and analgesic activities will be evaluated in further studies.

\section{Conclusions}

In the present study we report the synthesis, characterization and anti-inflammatory/analgesic evaluation of thiazolo[3,2-b][1,2,4]triazole derivatives and their corresponding acyclic intermediates, bearing benzenesulfonamide moiety. The structures of the new compounds were confirmed by spectral analysis (IR, ${ }^{1} \mathrm{H}$ NMR, ${ }^{13} \mathrm{C}$ NMR and MS). In pharmacological studies, the anti-inflammatory and analgesic activities of the synthesized compounds were assessed in vivo using a pharmacological model of acute inflammation induced in rats. Compounds $\mathbf{6 d}, \mathbf{5 c}$, and $\mathbf{5 b}$ showed the most significant anti-inflammatory activity at 2 hours, 3 hours, respectively 4 hours after the induction of inflammation. Compounds 5a, 5b, 5c, 6a and $\mathbf{6 d}$ presented significant analgesic properties at 4 hours 
after inflammation occurred. All compounds showed significant lower ulcerogenic risk than the reference drug, diclofenac. These compounds are good candidates for further investigations regarding their potential use in therapy for the treatment of pain and inflammatory diseases.

\section{Acknowledgement}

This work was possible with the financial support of "Iuliu Haţieganu" University of Medicine and Pharmacy, Cluj-Napoca, Romania through the internal research grant no. 5200/32/01.03.2017.

\section{Conflicts of Interest}

The authors declare that they have no conflict of interest.

\section{References}

1. Ahmadi F, Ghayahbashi MR, Sharifzadeh M, Alipoiur E, Ostad SN, Vosooghi M, Khademi HR, Amini M, Synthesis and evaluation of antiinflammatory and analgesic activities of new 1,2,4triazole derivatives. Med Chem., 2014; 11(1): 6976.

2. Aktay G, Tozkoparan B, Ertan M, Investigation of antioxidant properties of some 6 -( $\alpha$-aminobenzyl $)$ thiazolo[3,2-b]-1,2,4-triazole-5-ol compounds. $J$ Enzyme Inhib Med Chem., 2009; 24(3): 898-902.

3. Anderson GD, Hauser SD, McGarity KL, Bremer ME, Isakson PC, Gregory SA, Selective inhibition of cyclooxygenase (COX)-2 reverses inflammation and expression of COX-2 and interleukin 6 in rat adjuvant arthritis. J Clin Invest., 1966; 97: 2672-2679.

4. Assarzadeh MJ, Almasirad A, Shafiee A, Koopaei MN, Abdollahi M, Synthesis of new thiazolo[3,2b] $[1,2,4]$ triazole- $6(5 \mathrm{H})$-one derivatives as potent analgesic and anti-inflammatory agents. Med Chem Res., 2014; 23: 948-957.

5. Barbuceanu SF, Almajan GL, Saramet I, Draghici C, Tarcomnicu AI, Bancescu G, Synthesis, characterization and evaluation of antibacterial activity of some thiazolo[3,2-b][1,2,4] triazole incorporating diphenylsulfone moieties. Eur J Med Chem., 2009; 44: 4752-4757.

6. Bhattacharyya S, Gill R, Chen ML, Zhang F, Linhardt RJ, Dudeja PK, Tobacman JK, Toll-like receptor 4 mediates induction of the Bcl10NFкарpaB-interleukin-8 inflammatory pathway by carrageenan in human intestinal epithelial cells. $J$ Biol Chem., 2008; 283: 10550-10558.

7. Buzás GM, Supuran CT, The history and rationale of using carbonic anhydrase inhibitors in the treatment of peptic ulcers. In memoriam Ioan Pușcaș (1932-2015). J Enzyme Inhib Med Chem., 2016; 31: 527-533.

8. Cioli V, Silvestrini B, Dordoni F, Evaluation of the potential of gastric ulceration after administration of certain drugs. Exp Mol Pathol., 1967; 6: 68-83.

9. Conea S, Mogosan C, Vostinaru O, Toma CC, Cuc Hepcal I, Cazacu I, Pop C, Vlase L, Polyphenolic profile, anti-inflammatory and antinociceptive activity of an extract from Arctium lappa L. roots. Not Bot Horti Agrobo., 2017; 45: 59-64.

10. Cristina A, Leonte D, Vlase L, Bencze LC, Imre S, Zaharia $\mathrm{V}$, Heterocycles 42. Synthesis and characterization of new thiazolo[3,2-b][1,2,4]triazole derivatives with anti-inflammatory potential. Farmacia, 2018; 66(1): 88-96.

11. Di Rosa M, Giroud JP, Willoughby DA, Studies of the mediators of the acute inflammatory response induced in rats in different sites by carrageenan and turpentine. J Pathol., 1971; 104: 15-29.

12. Doğdaş E, Tozkoparan B, Kaynak FB, Eriksson L, Küpeli E, Yeşilada E, Ertan M, Design and synthesis of some new thiazolo [3,2-b]-1,2,4-triazole-5(6H)ones substituted with flurbiprofen as anti-inflammatory and analgesic agents. Arzneimittelforschung, 2007; 57: 196-202.

13. Flower RJ, The development of COX2 inhibitors. Nat Rev Drug Discov., 2003; 2: 179-191.

14. Gadad AK, Palkar MB, Anand K, Noolvi MN, Boreddy TS, Wagwade J, Synthesis and biological evaluation of 2-trifluoromethyl/sulfonamido-5,6diaryl substituted imidazo[2,1-b]-1,3,4-thiadiazoles: A novel class of cyclooxygenase-2 inhibitors. Bioorg Med Chem., 2008; 16: 276-283.

15. Gassani BC, Rezende RM, Paiva-Lima P, FerreiraAlves DL, dos Reis WG, Bakhle YS, de Francischi $\mathrm{JN}$, Is the sulphonamide radical in the celecoxib molecule essential for its analgesic activity?. Pharmacol Res., 2010; 62: 439-443.

16. Karin M, Ben-Neriah Y, Phosphorylation meets ubiquitination: The control of NF-kB activity. Annu Rev Immunol., 2000; 18: 621-663.

17. Karthikeyan MS, Synthesis, analgesic, anti-inflammatory and antimicrobial studies of 2,4-dichloro-5-fluorophenyl containing thiazolotriazoles. Eur J Med Chem., 2009; 44: 827-833.

18. Kumar R, Bua S, Ram S, Del Prete S, Capasso C, Supuran CT, Sharma PK, Benzenesulfonamide bearing imidazothiadiazole and thiazolotriazole scaffolds as potent tumor associated human carbonic anhydrase IX and XII inhibitors. Bioorg Med Chem., 2017; 25: 1286-1293.

19. Kurumbail RG, Stevens AM, Gierse JK, McDonald JJ, Stegeman RA, Pak JY, Gildehaus D, Miyashiro JM, Penning TD, Seibert K, Isakson PC, Stallings WC, Structural basis for selective inhibition of cyclooxygenase- 2 by anti-inflammatory agents. Nature, 1996; 384: 644-648.

20. Lesyk R, Vladzimirska O, Holota S, Zaprutko L, Gzella A, New 5-substituted thiazolo[3,2-b][1,2,4] triazol-6-ones: Synthesis and anticancer evaluation. Eur J Med Chem., 2007; 42: 641-648.

21. Mehta D, Bhargava D, Non-steroidal anti-inflammatory drugs and gastrointestinal toxicity. Apollo Med., 2010; 7: 251-262.

22. Micklewright R, Lane S, Linley W, McQuade C, Thompson F, Maskrey N, NSAIDs, gastroprotection and cyclo-oxygenase-II-selective inhibitors. Aliment Pharmacol Ther., 2003; 17: 321-332.

23. Mogosan C, Vostinaru O, Oprean R, Heghes C, Filip L, Balica G, Moldovan RI, A comparative analysis of the chemical composition, anti-inflammatory, and antinociceptive effects of the essential oils from 
three species of Mentha cultivated in Romania. Molecules, 2017; 22: 263.

24. Mogoșan C, Voștinaru O, Pârvu AE, Pop C, Zaharia V, An evaluation of the anti-inflammatory potential of some polyheterocyclic compounds with thiazole rings in acute inflammation models. Part I. Vascular response. Farmacia, 2013; 61(2): 323-329.

25. Morris C.J. Carrageenan-induced paw edema in the rat and mouse. Methods Mol Biol., 2003; 225: 115-122.

26. Narayana B, Vijaya Raj KK, Ashalatha BV, Kumari NS, Sarojini BK, Synthesis of some new 5-(2substituted-1,3-thiazol-5-yl)-2-hydroxy benzamides and their 2-alkoxy derivatives as possible antifungal agents. Eur J Med Chem., 2004; 39: 867-872.

27. Pârvu AE, Mogoșan C, Voștinaru O, Pop C, Zaharia $\mathrm{V}$, Evaluation of the anti-inflammatory potential of some polyheterocyclic compounds with thiazole ring in acute inflammation models. Part II. Cellular response. Farmacia, 2013; 61(3): 591-597.

28. Pignatello R, Mazzone S, Panico AM, Mazzone G, Pennisi G, Castana R, Matera M, Blandino G, Synthesis and biological evaluation of thiazolo-triazole derivatives. Eur J Med Chem., 1991; 2: 929-938.

29. Plech T, Kaproń B, Łuszczki J, Wujec M, Paneth A, Siwek A, Kołaczkowski M, Zołnierek M, Nowak G, Studies on the anticonvulsant activity and influence on GABA-ergic neurotransmission of 1,2,4-triazole-3thione-based compounds. Molecules, 2014; 19: 1127911299.

30. Randall LO, Selitto JJ, A method for measurement of analgesic activity on inflamed tissue. Arch Int Pharmacodyn Ther., 1957; 111: 409-419.

31. Rao P, Knaus EE, Evolution of Nonsteroidal AntiInflammatory Drugs (NSAIDs): Cyclooxygenase (COX) Inhibition and Beyond. J Pharm Pharm Sci., 2008; 11: 81s-110s.

32. Rostom SA, El-Ashmawy I, Abd El Razik H, Badr M, Ashour HM, Design and synthesis of some thiazolyl and thiadiazolyl derivatives of antipyrine as potential non-acidic anti-inflammatory, analgesic and antimicrobial agents. Bioorg Med Chem., 2009; 17: 882-895.

33. Sahu JK, Ganguly S, Kaushik A, Triazoles: A valuable insight into recent developments and biological activities. Chin J Nat Med., 2013; 11: 456-465.
34. Sancak K, Ünver Y, Ünlüer D, Düğdü E, Kör G, Çelik F, Birinci E, Synthesis, characterization, and antioxidant activities of new trisubstituted triazoles. Turk J Chem., 2012; 36: 457-466.

35. Simiti I, Zaharia V, Coman M, Demian H, Heterocyclen 65. Darstellung und chemisches verhalten einiger 2-aryl-6-ethoxyxarbonyl-5-methyl-thiazolo[3,2-b]1,2,4-triazole. Arch Pharm., 1991; 324: 49-51.

36. Simmons DL, Cyclooxygenase isozymes: The biology of prostaglandin synthesis and inhibition. Pharmacol Rev., 2004; 56: 387-437.

37. Somani R, Chavan A, Makhija D, Synthesis and pharmacological evaluation of anti-inflammatory mutual amide prodrugs. Ind J Pharm Sci., 2013; 75: 353-357.

38. Toma A, Mogosan C, Vlase L, Leonte D, Zaharia $\mathrm{V}$, Heterocycles 39. Synthesis, characterization and evaluation of the anti-inflammatory activity of thiazolo [3,2-b][1,2,4]triazole derivatives bearing pyridin3/4-yl moiety. Med Chem Res., 2017; 26: 2602-2613.

39. Tozkoparan B, Aytaç SP, Gürsoy Ş, Aktay G, Design and synthesis of some thiazolotriazolyl esters as anti-inflammatory and analgesic agents. Med Chem Res., 2012; 21: 192-201.

40. Vinegar R, Schreiber W, Hugo R, Biphasic development of carrageenin edema in rats. $J$ Pharmacol Exp Ther., 1969; 166: 96-103.

41. Vinegar R, Truax JF, Selph JL, Johnston PR, Venable AL, McKenzie KK, Pathway to carrageenan-induced inflammation in the hind limb of the rat. Fed Proc., 1987; 46: 118-126.

42. Winter CA, Risley EA, Nuss GW, Carrageenin-induced edema in hind paw of the rat as an assay for antiinflammatory drugs. Proc Soc Exp Biol Med.. 1962; 111: 544-547.

43. Zaharia V, Imre S, Palibroda N, Heterocycles. Obtaining and physico-chemical characterization of some thiazolo and thiazolo[3,2-b][1,2,4]triazolic hydroxy-heterochalcones. Rev Chim., 2009; 60: 391-397.

44. Zaharia V, Teodor F, Kory M, Sandor V, Simiti I, Heterocycles 66. Obtention and anti-inflammatory action of some 2-aryl-5-R1-6R2-thiazolo[3,2-b]1,2,4-triazoles. Clujul Med., 1990; LXIII: 69-75. 